TAIWANESE JOURNAL OF MATHEMATICS

Vol. 14, No. 4, pp. 1635-1646, August 2010

This paper is available online at http://www.tjm.nsysu.edu.tw/

\title{
A UNIFIED GENERALIZATION OF ACZÉL, POPOVICIU AND BELLMAN'S INEQUALITIES
}

\author{
Shanhe Wu
}

\begin{abstract}
In this paper, we give a unified generalization of Aczél, Popoviciu and Bellman's inequalities. The result is then applied to deriving a refinement of Aczél's inequality and Bellman's inequality. As consequences, several interesting integral inequalities of Aczél-Popoviciu-Bellman type are obtained.
\end{abstract}

\section{INTRODUCTION}

Aczél [1] proved the following result:

$$
\left(a_{1}^{2}-\sum_{i=2}^{n} a_{i}^{2}\right)\left(b_{1}^{2}-\sum_{i=2}^{n} b_{i}^{2}\right) \leq\left(a_{1} b_{1}-\sum_{i=2}^{n} a_{i} b_{i}\right)^{2},
$$

where $a_{i}, b_{i}(i=1,2, \ldots, n)$ are real numbers such that $a_{1}^{2}-\sum_{i=2}^{n} a_{i}^{2}>0$ or $b_{1}^{2}-\sum_{i=2}^{n} b_{i}^{2}>0$. This inequality is known in the literature as Aczél's inequality (see Mitrinović and Vasić [2]).

Popoviciu [3] generalized inequality (1) in the following form:

$$
\left(a_{1}^{p}-\sum_{i=2}^{n} a_{i}^{p}\right)\left(b_{1}^{p}-\sum_{i=2}^{n} b_{i}^{p}\right) \leq\left(a_{1} b_{1}-\sum_{i=2}^{n} a_{i} b_{i}\right)^{p},
$$

where $p \geq 1, a_{i}, b_{i}(i=1,2, \ldots, n)$ are nonnegative real numbers such that $a_{1}^{p}-\sum_{i=2}^{n} a_{i}^{p}>0$ or $b_{1}^{p}-\sum_{i=2}^{n} b_{i}^{p}>0$.

Received October 23, 2008, accepted January 4, 2009.

Communicated by Sen-Yen Shaw.

2000 Mathematics Subject Classification: 26D15, 26D20.

Key words and phrases: Acz él's inequality, Popoviciu's inequality, Bellman's inequality, Power mean, Generalization, Refinement. 
However, there is an error in Popoviciu's result. Bjelica [4], Losonczi and Páles [5] showed via counterexamples that the inequality (2) is not true in general for $p>2$, and indicated that the inequality (2) holds true under the condition that $0<p \leq 2, a_{1}^{p}-\sum_{i=2}^{n} a_{i}^{p}>0$ and $b_{1}^{p}-\sum_{i=2}^{n} b_{i}^{p}>0$.

Bellman [6] presented an analogue of Aczél-Popoviciu inequality, as follows

$$
\left(a_{1}^{p}-\sum_{i=2}^{n} a_{i}^{p}\right)^{\frac{1}{p}}+\left(b_{1}^{p}-\sum_{i=2}^{n} b_{i}^{p}\right)^{\frac{1}{p}} \leq\left(\left(a_{1}+b_{1}\right)^{p}-\sum_{i=2}^{n}\left(a_{i}+b_{i}\right)^{p}\right)^{\frac{1}{p}}
$$

where $p \geq 1, a_{i}, b_{i}(i=1,2, \cdots, n)$ are positive numbers such that $a_{1}^{p}-\sum_{i=2}^{n} a_{i}^{p}>0$ and $b_{1}^{p}-\sum_{i=2}^{n} b_{i}^{p}>0$.

Aczél, Popoviciu and Bellman's inequalities have important applications in the theory of functional equations in non-Euclidean geometry. Due to the importance of these inequalities, they have been given considerable attention by mathematicians. A comprehensive survey on these inequalities can be found in the monograph [7, p. 117]. During the past few years, numerous generalizations, improvements and variants of Aczél's inequality and Popoviciu's inequality have appeared in the literature, see Mascioni [8], Mercer [9], Sun [10], Dragomir and Mond [11], Wu and Debnath [12, 13], Wu [14-17] and Cho et al. [18].

The purpose of this paper is to establish a unified generalization of Aczél, Popoviciu and Bellman's inequalities. We next provide an application of the obtained result to the refinements of Popoviciu's inequality and Bellman's inequality. Finally, in Section 4 we give several interesting integral inequalities of AczélPopoviciu-Bellman type.

\section{LEMMAS}

In order to prove our main results, we need the following lemmas.

Lemma 1. (Generalized Minkowski's inequality [19]). Let $x_{i j}>0(i=1,2, \ldots$, $n, j=1,2, \ldots, m)$ and $0<p \leq 1$. Then

$$
\left[\sum_{i=1}^{n}\left(\sum_{j=1}^{m} x_{i j}\right)^{\frac{1}{p}}\right]^{p} \leq \sum_{j=1}^{m}\left(\sum_{i=1}^{n} x_{i j}^{\frac{1}{p}}\right)^{p}
$$

with equality holding if and only if $p=1$, or $\frac{x_{1 j}}{x_{11}}=\frac{x_{2 j}}{x_{21}}=\cdots=\frac{x_{n j}}{x_{n 1}}(j=$ $2,3, \ldots, m)$ for $0<p<1$. Furthermore, the inequality (4) is reversed for $p>1$. 
Lemma 2. (Hölder's inequality [19]). Let $x_{i j}>0, p_{j}>0(i=1,2, \ldots, n, j=$ $1,2, \ldots, m)$ and $p_{1}+p_{2}+\cdots+p_{m}=1$. Then

$$
\prod_{j=1}^{m}\left(\sum_{i=1}^{n} x_{i j}\right)^{p_{j}} \geq \sum_{i=1}^{n} \prod_{j=1}^{m} x_{i j}^{p_{j}}
$$

with equality holding if and only if $\frac{x_{1 j}}{x_{11}}=\frac{x_{2 j}}{x_{21}}=\cdots=\frac{x_{n j}}{x_{n 1}}(j=2,3, \ldots, m)$.

Lemma 3. Let $x_{i}(i=1,2, \ldots, n)$ be positive real numbers such that $x_{1}-$ $x_{2}-\cdots-x_{n}>0$, and let $p \leq 1$. Then

$$
x_{1}^{p}-\sum_{i=2}^{n} x_{i}^{p} \leq\left(x_{1}-\sum_{i=2}^{n} x_{i}\right)^{p}
$$

with equality holding if and only if $p=1$.

Proof. From the hypotheses: $p-1 \leq 0, x_{1}>x_{2}+\cdots+x_{n}$, we deduce that

$$
\begin{aligned}
\left(x_{1}-\sum_{i=2}^{n} x_{i}\right)^{p}+\sum_{i=2}^{n} x_{i}^{p} & =\left(x_{1}-\sum_{i=2}^{n} x_{i}\right)\left(x_{1}-\sum_{i=2}^{n} x_{i}\right)^{p-1}+\sum_{i=2}^{n} x_{i} x_{i}^{p-1} \\
& \geq\left(x_{1}-\sum_{i=2}^{n} x_{i}\right) x_{1}^{p-1}+\sum_{i=2}^{n} x_{i} x_{1}^{p-1} \\
& =x_{1}^{p} .
\end{aligned}
$$

Lemma 3 is proved.

3. Generalizations of Aczél, Popoviciu and Bellman's Inequalities

As in [2], the power mean of order $r$ for positive numbers $x_{1}, x_{2}, \ldots, x_{m}$ is defined by

$$
M_{m}^{[r]}\left(x_{1}, x_{2}, \ldots, x_{m}\right)= \begin{cases}\left(\frac{x_{1}^{r}+x_{2}^{r}+\cdots+x_{m}^{r}}{m}\right)^{\frac{1}{r}} & \text { for } \quad r \neq 0, \\ \left(x_{1} x_{2} \cdots x_{m}\right)^{\frac{1}{m}} & \text { for } \quad r=0 .\end{cases}
$$

We start this section by establishing the following combined generalization of Aczél, Popoviciu and Bellman's inequalities:

Theorem 1. Let $p \geq r \geq 0, p \neq 0, a_{i j}>0, a_{1 j}^{p}-\sum_{i=2}^{n} a_{i j}^{p}>0(i=1,2, \ldots, n$, $j=1,2, \ldots, m)$, and let $\widetilde{a}_{j}=\left(a_{1 j}^{p}-\sum_{i=2}^{n} a_{i j}^{p}\right)^{1 / p}(j=1,2, \ldots, m)$. Then the 
following inequality holds

(7) $\left(M_{m}^{[r]}\left(\widetilde{a}_{1}, \ldots, \widetilde{a}_{m}\right)\right)^{p} \leq\left(M_{m}^{[r]}\left(a_{11}, \ldots, a_{1 m}\right)\right)^{p}-\sum_{i=2}^{n}\left(M_{m}^{[r]}\left(a_{i 1}, \ldots, a_{i m}\right)\right)^{p}$.

Equality holds in (7) if and only if $p=r \neq 0$, or $\frac{a_{1 j}}{a_{11}}=\frac{a_{2 j}}{a_{21}}=\cdots=\frac{a_{n j}}{a_{n 1}}$ $(j=2,3, \ldots, m)$ for $p>r$.

Proof. We consider the following two cases.

Case (I). When $r>0$. It is easy to see that the inequality (7) is equivalent to the following inequality:

$$
\left(\sum_{j=1}^{m}\left(a_{1 j}^{p}-\sum_{i=2}^{n} a_{i j}^{p}\right)^{\frac{r}{p}}\right)^{\frac{p}{r}} \leq\left(\sum_{j=1}^{m} a_{1 j}^{r}\right)^{\frac{p}{r}}-\sum_{i=2}^{n}\left(\sum_{j=1}^{m} a_{i j}^{r}\right)^{\frac{p}{r}} .
$$

Using the generalized Minkowski's inequality with $0<r / p \leq 1$ gives

$$
\left(\sum_{i=2}^{n}\left(\sum_{j=1}^{m} a_{i j}^{r}\right)^{\frac{p}{r}}\right)^{\frac{r}{p}} \leq \sum_{j=1}^{m}\left(\sum_{i=2}^{n}\left(a_{i j}^{r}\right)^{\frac{p}{r}}\right)^{\frac{r}{p}}
$$

that is,

$$
\sum_{i=2}^{n}\left(\sum_{j=1}^{m} a_{i j}^{r}\right)^{\frac{p}{r}} \leq\left(\sum_{j=1}^{m}\left(\sum_{i=2}^{n} a_{i j}^{p}\right)^{\frac{r}{p}}\right)^{\frac{p}{r}}
$$

Thus, we have

(9)

$$
\begin{aligned}
& \left(\sum_{j=1}^{m}\left(a_{1 j}^{p}-\sum_{i=2}^{n} a_{i j}^{p}\right)^{\frac{r}{p}}\right)^{\frac{p}{r}}+\sum_{i=2}^{n}\left(\sum_{j=1}^{m} a_{i j}^{r}\right)^{\frac{p}{r}} \\
\leq & \left(\sum_{j=1}^{m}\left(a_{1 j}^{p}-\sum_{i=2}^{n} a_{i j}^{p}\right)^{\frac{r}{p}}\right)^{\frac{p}{r}}+\left(\sum_{j=1}^{m}\left(\sum_{i=2}^{n} a_{i j}^{p}\right)^{\frac{r}{p}}\right)^{\frac{p}{r}} .
\end{aligned}
$$

Now, using the generalized Minkowski's inequality with $p / r \geq 1$, it follows that

(10) $\left(\sum_{j=1}^{m}\left(a_{1 j}^{p}-\sum_{i=2}^{n} a_{i j}^{p}\right)^{\frac{r}{p}}\right)^{\frac{p}{r}}+\left(\sum_{j=1}^{m}\left(\sum_{i=2}^{n} a_{i j}^{p}\right)^{\frac{r}{p}}\right)^{\frac{p}{r}} \leq\left(\sum_{j=1}^{m}\left(a_{1 j}^{p}\right)^{\frac{r}{p}}\right)^{\frac{p}{r}}$. 
By combining inequalities (9) and (10), we obtain

$$
\left(\sum_{j=1}^{m}\left(a_{1 j}^{p}-\sum_{i=2}^{n} a_{i j}^{p}\right)^{\frac{r}{p}}\right)^{\frac{p}{r}}+\sum_{i=2}^{n}\left(\sum_{j=1}^{m} a_{i j}^{r}\right)^{\frac{p}{r}} \leq\left(\sum_{j=1}^{m} a_{1 j}^{r}\right)^{\frac{p}{r}}
$$

which is the required inequality (8). This proves the inequality (7) for the case of $r>0$.

Case (II). When $r=0$. The inequality (7) can be rewritten as

$$
\prod_{j=1}^{m}\left(a_{1 j}^{p}-\sum_{i=2}^{n} a_{i j}^{p}\right)^{\frac{1}{m}} \leq \prod_{j=1}^{m} a_{1 j}^{\frac{p}{m}}-\sum_{i=2}^{n} \prod_{j=1}^{m} a_{i j}^{\frac{p}{m}} .
$$

Applying the Hölder's inequality gives

$$
\prod_{j=1}^{m} a_{1 j}^{\frac{p}{m}}=\prod_{j=1}^{m}\left(\left(a_{1 j}^{p}-\sum_{i=2}^{n} a_{i j}^{p}\right)+\sum_{i=2}^{n} a_{i j}^{p}\right)^{\frac{1}{m}} \geq \prod_{j=1}^{m}\left(a_{1 j}^{p}-\sum_{i=2}^{n} a_{i j}^{p}\right)^{\frac{1}{m}}+\sum_{i=2}^{n} \prod_{j=1}^{m} a_{i j}^{\frac{p}{m}},
$$

which implies the desired inequality (11). This proves the inequality (7) for the case of $r=0$.

From Lemmas 1 and 2 we can easily deduce that the equality holds in (7) if and only if $p=r \neq 0$, or $\frac{a_{1 j}}{a_{11}}=\frac{a_{2 j}}{a_{21}}=\cdots=\frac{a_{n j}}{a_{n 1}}(j=2,3, \ldots, m)$ for $p>r$. The proof of Theorem 1 is complete.

In the following we will not discuss the conditions for equality because they can be obtained directly from Theorem 1 .

Remark 1. Putting $r=1$ in Theorem 1 gives the following generalization of Bellman's inequality:

Corollary 1. Let $p \geq 1, a_{i j}>0, a_{1 j}^{p}-\sum_{i=2}^{n} a_{i j}^{p}>0(i=1,2, \ldots, n, j=$ $1,2, \ldots, m)$. Then we have the inequality

$$
\sum_{j=1}^{m}\left(a_{1 j}^{p}-\sum_{i=2}^{n} a_{i j}^{p}\right)^{\frac{1}{p}} \leq\left(\left(\sum_{j=1}^{m} a_{1 j}\right)^{p}-\sum_{i=2}^{n}\left(\sum_{j=1}^{m} a_{i j}\right)^{p}\right)^{\frac{1}{p}} .
$$

Putting $r=0$ in Theorem 1 and making use of Lemma 3, a generalization of Aczél's inequality is derived as follows:

Corollary 2. Let $m \geq p>0, a_{i j}>0, a_{1 j}^{p}-\sum_{i=2}^{n} a_{i j}^{p}>0(i=1,2, \ldots, n$, $j=1,2, \ldots, m)$. Then we have the inequality 


$$
\prod_{j=1}^{m}\left(a_{1 j}^{p}-\sum_{i=2}^{n} a_{i j}^{p}\right) \leq\left(\prod_{j=1}^{m} a_{1 j}-\sum_{i=2}^{n} \prod_{j=1}^{m} a_{i j}\right)^{p}
$$

Remark 2. In a special case when $m=2$, inequality (12) reduces to Bellman's inequality (3).

In Corollary 2, setting $m=2, a_{i 1}=a_{i}, a_{i 2}=b_{i}(i=1,2, \ldots, n)$, we obtain a modified version of Popoviciu's inequality (2), i.e.,

Corollary 3. Let $2 \geq p>0$, and let $a_{i}, b_{i}(i=1,2, \ldots, n)$ be positive real numbers such that $a_{1}^{p}-\sum_{i=2}^{n} a_{i}^{p}>0$ and $b_{1}^{p}-\sum_{i=2}^{n} b_{i}^{p}>0$. Then

$$
\left(a_{1}^{p}-\sum_{i=2}^{n} a_{i}^{p}\right)\left(b_{1}^{p}-\sum_{i=2}^{n} b_{i}^{p}\right) \leq\left(a_{1} b_{1}-\sum_{i=2}^{n} a_{i} b_{i}\right)^{p} .
$$

In the next result, we establish seveval refinements of the generalized Aczel's inequality and Bellman's inequality.

Theorem 2. Let $m \geq p>0, a_{i j}>0, a_{1 j}^{p}-\sum_{i=2}^{n} a_{i j}^{p}>0(i=1,2, \ldots, n$, $j=1,2, \ldots, m)$. Then, for $1<k<n$ we have the inequality

$$
\prod_{j=1}^{m}\left(a_{1 j}^{p}-\sum_{i=2}^{n} a_{i j}^{p}\right) \leq R\left(a_{11}, \ldots, a_{n m}\right) \leq\left(\prod_{j=1}^{m} a_{1 j}-\sum_{i=2}^{n} \prod_{j=1}^{m} a_{i j}\right)^{p}
$$

where

$$
R\left(a_{11}, \ldots, a_{n m}\right)=\left[\prod_{j=1}^{m}\left(a_{1 j}^{p}-\sum_{i=2}^{k} a_{i j}^{p}\right)^{\frac{1}{p}}-\sum_{i=k+1}^{n} \prod_{j=1}^{m} a_{i j}\right]^{p} .
$$

Proof. By applying Corollary 2, we have

$$
\begin{aligned}
& \prod_{j=1}^{m}\left(a_{1 j}^{p}-\sum_{i=2}^{n} a_{i j}^{p}\right)=\prod_{j=1}^{m}\left(\left(a_{1 j}^{p}-\sum_{i=2}^{k} a_{i j}^{p}\right)-\sum_{i=k+1}^{n} a_{i j}^{p}\right) \\
\leq & {\left[\prod_{j=1}^{m}\left(a_{1 j}^{p}-\sum_{i=2}^{k} a_{i j}^{p}\right)^{\frac{1}{p}}-\sum_{i=k+1}^{n} \prod_{j=1}^{m} a_{i j}\right]^{p} }
\end{aligned}
$$


and

$$
\prod_{j=1}^{m}\left(a_{1 j}^{p}-\sum_{i=2}^{k} a_{i j}^{p}\right)^{\frac{1}{p}} \leq \prod_{j=1}^{m} a_{1 j}-\sum_{i=2}^{k} \prod_{j=1}^{m} a_{i j} .
$$

Combining inequalities (16) and (17) leads to the desired inequality (15). Theorem 2 is proved.

Theorem 3. Let $p \geq 1, a_{i j}>0, a_{1 j}^{p}-\sum_{i=2}^{n} a_{i j}^{p}>0(i=1,2, \ldots, n, j=$ $1,2, \ldots, m)$. Then, for $1<k<n$ we have the inequality

$$
\begin{aligned}
& \sum_{j=1}^{m}\left(a_{1 j}^{p}-\sum_{i=2}^{n} a_{i j}^{p}\right)^{\frac{1}{p}} \leq Q\left(a_{11}, \ldots, a_{n m}\right) \\
\leq & \left(\left(\sum_{j=1}^{m} a_{1 j}\right)^{p}-\sum_{i=2}^{n}\left(\sum_{j=1}^{m} a_{i j}\right)^{p}\right)^{\frac{1}{p}},
\end{aligned}
$$

where

$$
Q\left(a_{11}, \ldots, a_{n m}\right)=\left[\left(\sum_{j=1}^{m}\left(a_{1 j}^{p}-\sum_{i=2}^{k} a_{i j}^{p}\right)^{\frac{1}{p}}\right)^{p}-\sum_{i=k+1}^{n}\left(\sum_{j=1}^{m} a_{i j}\right)^{p}\right]^{\frac{1}{p}} .
$$

Proof. By applying Corollary 1, we have

$$
\begin{aligned}
& \sum_{j=1}^{m}\left(a_{1 j}^{p}-\sum_{i=2}^{n} a_{i j}^{p}\right)^{\frac{1}{p}}=\sum_{j=1}^{m}\left(\left(a_{1 j}^{p}-\sum_{i=2}^{k} a_{i j}^{p}\right)-\sum_{i=k+1}^{n} a_{i j}^{p}\right)^{\frac{1}{p}} \\
\leq & {\left[\left(\sum_{j=1}^{m}\left(a_{1 j}^{p}-\sum_{i=2}^{k} a_{i j}^{p}\right)^{\frac{1}{p}}\right)^{p}-\sum_{i=k+1}^{n}\left(\sum_{j=1}^{m} a_{i j}\right)^{p}\right]^{\frac{1}{p}} }
\end{aligned}
$$

and

$$
\left(\sum_{j=1}^{m}\left(a_{1 j}^{p}-\sum_{i=2}^{k} a_{i j}^{p}\right)^{\frac{1}{p}}\right)^{p} \leq\left(\sum_{j=1}^{m} a_{1 j}\right)^{p}-\sum_{i=2}^{k}\left(\sum_{j=1}^{m} a_{i j}\right)^{p} .
$$

The proof of Theorem 3 is completed by combining the inequalities (19) and (20). 
Remark 3. As a direct consequence of Theorem 2 and Theorem 3, setting $m=2, a_{i 1}=a_{i}, a_{i 2}=b_{i}(i=1,2, \ldots, n)$ in (15) and (18), respectively, yields

Corollary 4. Let $2 \geq p>0$, and let $a_{i}, b_{i}(i=1,2, \ldots, n)$ be positive real numbers such that $a_{1}^{p}-\sum_{i=2}^{n} a_{i}^{p}>0$ and $b_{1}^{p}-\sum_{i=2}^{n} b_{i}^{p}>0$. Then, for $1<k<n$ we have the inequality

$$
\left(a_{1}^{p}-\sum_{i=2}^{n} a_{i}^{p}\right)\left(b_{1}^{p}-\sum_{i=2}^{n} b_{i}^{p}\right) \leq R\left(a_{1}, b_{1}, \ldots, a_{n}, b_{n}\right) \leq\left(a_{1} b_{1}-\sum_{i=2}^{n} a_{i} b_{i}\right)^{p},
$$

where

$$
R\left(a_{1}, b_{1}, \ldots, a_{n}, b_{n}\right)=\left[\left(a_{1}^{p}-\sum_{i=2}^{k} a_{i}^{p}\right)^{\frac{1}{p}}\left(b_{1}^{p}-\sum_{i=2}^{k} b_{i}^{p}\right)^{\frac{1}{p}}-\sum_{i=k+1}^{n} a_{i} b_{i}\right]^{p} .
$$

Corollary 5. Let $p \geq 1$, and let $a_{i}, b_{i}(i=1,2, \ldots, n)$ be positive real numbers such that $a_{1}^{p}-\sum_{i=2}^{n} a_{i}^{p}>0$ and $b_{1}^{p}-\sum_{i=2}^{n} b_{i}^{p}>0$. Then, for $1<k<n$ we have the inequality

$$
\begin{aligned}
& \left(a_{1}^{p}-\sum_{i=2}^{n} a_{i}^{p}\right)^{\frac{1}{p}}+\left(b_{1}^{p}-\sum_{i=2}^{n} b_{i}^{p}\right)^{\frac{1}{p}} \\
\leq & Q\left(a_{1}, b_{1}, \ldots, a_{n}, b_{n}\right) \leq\left(\left(a_{1}+b_{1}\right)^{p}-\sum_{i=2}^{n}\left(a_{i}+b_{i}\right)^{p}\right)^{\frac{1}{p}},
\end{aligned}
$$

where

$Q\left(a_{1}, b_{1}, \ldots, a_{n}, b_{n}\right)=\left[\left(\left(a_{1}^{p}-\sum_{i=2}^{k} a_{i}^{p}\right)^{\frac{1}{p}}+\left(b_{1}^{p}-\sum_{i=2}^{k} b_{i}^{p}\right)^{\frac{1}{p}}\right)^{p}-\sum_{i=k+1}^{n}\left(a_{i}+b_{i}\right)^{p}\right]^{\frac{1}{p}}$.

Remark 4. The inequality (21) was proved by Díaz-Barrero et al. in a recent paper [20]. However, there is an error on the domain of the variable $p$. Namely, the authors claimed that the inequality (20) holds for any $p \in \mathbb{Z}^{+}$( $\mathbb{Z}^{+}$denotes the set of positive integers ). The assertion is clearly false because Popoviciu's inequality (2) is true only for $0<p \leq 2$ (see the introduction in Section 1).

\section{INTEGRAL VERSION OF ACZÉL-POPOVICIU-BELLMAN TYPE INEQUALITY}

In this section we provide several interesting integral inequalities of AczélPopoviciu-Bellman type. 
Theorem 4. Let $p \geq r \geq 0, p \neq 0, A_{j}>0(j=1,2, \ldots, m)$, let $f_{j}$ be positive Riemann integrable functions on $[a, b]$ such that $A_{j}^{p}-\int_{a}^{b} f_{j}^{p}(x) d x>0$ for all $j=1,2, \ldots, m$, and let $\widetilde{A}_{j}=\left(A_{j}^{p}-\int_{a}^{b} f_{j}^{p}(x) d x\right)^{1 / p}$. Then the following inequality holds

$$
\begin{aligned}
& \left(M_{m}^{[r]}\left(\widetilde{A}_{1}, \ldots, \widetilde{A}_{m}\right)\right)^{p} \leq\left(M_{m}^{[r]}\left(A_{1}, \ldots, A_{m}\right)\right)^{p} \\
- & \int_{a}^{b}\left(M_{m}^{[r]}\left(f_{1}(x), \ldots, f_{m}(x)\right)\right)^{p} d x .
\end{aligned}
$$

Proof. For any positive integer $n$, we choose an equidistant partition of $[a, b]$ as

$$
\begin{gathered}
a<a+\frac{b-a}{n}<\cdots<a+\frac{b-a}{n} i<\cdots<a+\frac{b-a}{n}(n-1)<b, \\
\Delta x_{i}=\frac{b-a}{n}, \quad i=1,2, \cdots, n .
\end{gathered}
$$

Since the hypothesis $A_{j}^{p}-\int_{a}^{b} f_{j}^{p}(x) d x>0(j=1,2, \ldots, m)$ implies that

$$
A_{j}^{p}-\lim _{n \rightarrow \infty} \sum_{i=1}^{n} f_{j}^{p}\left(a+\frac{i(b-a)}{n}\right) \frac{b-a}{n}>0 \quad(j=1,2, \ldots, m),
$$

there exists a positive integer $N$ such that

$$
A_{j}^{p}-\sum_{i=1}^{n} f_{j}^{p}\left(a+\frac{i(b-a)}{n}\right) \frac{b-a}{n}>0 \text { for all } n>N \text { and } j=1,2, \ldots, m \text {. }
$$

Applying Theorem 1, one obtains the following inequalities:

$$
\begin{aligned}
& {\left[\sum_{j=1}^{m}\left(A_{j}^{p}-\sum_{i=1}^{n} f_{j}^{p}\left(a+\frac{i(b-a)}{n}\right) \frac{b-a}{n}\right)^{\frac{r}{p}}\right]^{\frac{p}{r}} } \\
\leq & \left(\sum_{j=1}^{m} A_{j}^{r}\right)^{\frac{p}{r}}-\sum_{i=1}^{n}\left(\sum_{j=1}^{m} f_{j}^{r}\left(a+\frac{i(b-a)}{n}\right)\right)^{\frac{p}{r}} \frac{b-a}{n}
\end{aligned}
$$

for any $n>N$ and $r>0$;

$$
\begin{aligned}
& {\left[\prod_{j=1}^{m}\left(A_{j}^{p}-\sum_{i=1}^{n} f_{j}^{p}\left(a+\frac{i(b-a)}{n}\right) \frac{b-a}{n}\right)^{\frac{1}{p}}\right]^{\frac{p}{m}} } \\
\leq & \left(\prod_{j=1}^{m} A_{j}\right)^{\frac{p}{m}}-\sum_{i=1}^{n}\left(\prod_{j=1}^{m} f_{j}\left(a+\frac{i(b-a)}{n}\right)\right)^{\frac{p}{m}} \frac{b-a}{n}
\end{aligned}
$$


for any $n>N$ and $r=0$.

In view of the hypotheses that $f_{j}(j=1,2, \ldots, m)$ are positive Riemann integrable functions on $[a, b]$, we conclude that $f_{j}^{p},\left(\sum_{j=1}^{m} f_{j}^{r}\right)^{p / r}$ and $\left(\prod_{j=1}^{m} f_{j}\right)^{p / m}$ are also integrable on $[a, b]$. Passing to the limit $n \rightarrow \infty$ on both sides of the above inequalities, we obtain that

$$
\begin{aligned}
& {\left[\sum_{j=1}^{m}\left(A_{j}^{p}-\int_{a}^{b} f_{j}^{p}(x) d x\right)^{\frac{r}{p}}\right]^{\frac{p}{r}} } \\
\leq & \left(\sum_{j=1}^{m} A_{j}^{r}\right)^{\frac{p}{r}}-\int_{a}^{b}\left(\sum_{j=1}^{m} f_{j}^{r}(x)\right)^{\frac{p}{r}} d x \quad(r>0)
\end{aligned}
$$

and

$$
\begin{aligned}
& {\left[\prod_{j=1}^{m}\left(A_{j}^{p}-\int_{a}^{b} f_{j}^{p}(x) d x\right)^{\frac{1}{p}}\right]^{\frac{p}{m}} } \\
\leq & \left(\prod_{j=1}^{m} A_{j}\right)^{\frac{p}{m}}-\int_{a}^{b}\left(\prod_{j=1}^{m} f_{j}(x)\right)^{\frac{p}{m}} d x \quad(r=0) .
\end{aligned}
$$

Combining inequalities (24) and (25) leads to the inequality (23) asserted by Theorem 4. This completes the proof of Theorem 4 .

Remark 5. Putting $r=1$ in Theorem 4, we get the following integral version of Bellman's inequality:

Corollary 6. Let $p \geq 1, A_{j}>0(j=1,2, \ldots, m)$, and let $f_{j}$ be positive Riemann integrable functions on $[a, b]$ such that $A_{j}^{p}-\int_{a}^{b} f_{j}^{p}(x) d x>0$ for all $j=1,2, \ldots, m$. Then

$$
\sum_{j=1}^{m}\left(A_{j}^{p}-\int_{a}^{b} f_{j}^{p}(x) d x\right)^{\frac{1}{p}} \leq\left(\left(\sum_{j=1}^{m} A_{j}\right)^{p}-\int_{a}^{b}\left(\sum_{j=1}^{m} f_{j}(x)\right)^{p} d x\right)^{\frac{1}{p}}
$$

Putting $r=0$ and $p=m$ in Theorem 4, the integral version of Aczél-Popoviciu inequality is derived as follows:

Corollary 7. Let $A_{j}>0(j=1,2, \ldots, m)$, and let $f_{j}$ be positive Riemann integrable functions on $[a, b]$ such that $A_{j}^{m}-\int_{a}^{b} f_{j}^{m}(x) d x>0$ for all $j=1,2, \ldots, m$. 
Then

$$
\prod_{j=1}^{m}\left(A_{j}^{m}-\int_{a}^{b} f_{j}^{m}(x) d x\right)^{\frac{1}{m}} \leq \prod_{j=1}^{m} A_{j}-\int_{a}^{b}\left(\prod_{j=1}^{m} f_{j}(x)\right) d x
$$

\section{ACKNOWLEDGMENTS}

The present investigation was supported, in part, by the Natural Science Foundation of Fujian province of China under grant No. S0850023 and, in part, by the Foundation of Scientific Research Project of Fujian Province Education Department of China under grant No. JA08231.

\section{REFERENCES}

1. J. Aczel, Some general methods in the theory of functional equations in one variable, New applications of functional equations, Uspehi. Mat. Nauk (N.S.) 11, 69(3) (1956), 3-68 (in Russian).

2. D. S. Mitrinović and P. M. Vasic, Analytic Inequalities, Springer-Verlag, New York, 1970.

3. T. Popoviciu, On an inequality, Gaz. Mat. Fiz. Ser. A, 11(64) (1959), 451-461 (in Romanian).

4. M. Bjelica, On inequalities for indefinite form, Anal. Numer. Theor. Approx., 19 (1990), 105-109.

5. L. Losonczi and Z. Páles, Inequalities for indefinite forms, J. Math. Anal. Appl., 205 (1997) 148-156.

6. R. Bellman, On an inequality concerning an indefinite form, Amer. Math. Monthly, 63(2) (1956), 108-109.

7. D. S. Mitrinovic, J. E. Pecaric and A. M. Fink, Classical and New Inequalities in Analysis, Kluwer Academic Publishers, Dordrecht, 1993.

8. V. Mascioni, A note on Aczél type inequalities, J. Inequal. Pure Appl. Math., 3(5) (2002), Article 69. pp. 1-5 (electronic).

9. A. M. Mercer, Extensions of popoviciu's inequality using a general method, $J$. Inequal. Pure Appl. Math., 4(1) (2003), Article 11, pp. 1-4 (electronic).

10. X. H. Sun, Aczél-Chebyshev type inequality for positive linear functions, J. Math. Anal. Appl., 245 (2000), 393-403.

11. S. S. Dragomir and B. Mond, Some inequalities of Aczél type for Gramians in inner product spaces, Nonlinear Funct. Anal. Appl., 6(3) (2001), 411-424. 
12. S. Wu and L. Debnath, Generalizations of Aczél's inequality and Popoviciu's inequality, Indian J. Pure Appl. Math., 36(2) (2005), 49-62.

13. S. Wu and L. Debnath, A new generalization of Aczél's inequality and its applications to an improvement of Bellman's inequality, Appl. Math. Lett., 21(6) (2008), 588-593.

14. S. Wu, A further generalization of Aczel's inequality and Popoviciu's inequality, Math. Inequal. Appl., 10(3) (2007), 565-573.

15. S. Wu, Improvement of Aczél's inequality and Popoviciu's inequality, J. Inequal. Appl., 2007 (2007), Articl ID 72173, pp. 1-9 (electronic).

16. S. Wu, A new sharpened and generalized version of Hölder's inequality and its applications, Appl. Math. Comput., 197 (2008), 708-714.

17. S. Wu, Some improvements of Aczél's inequality and Popoviciu's inequality, Comput. Math. Appl., 56(5) (2008), 1196-1205.

18. Y. J. Cho, M. Matić and J. Pečarić, Improvements of some inequalities of Aczel's type, J. Math. Anal. Appl., 259 (2001), 226-240.

19. E. F. Beckenbach and R. Bellman, Inequalities, Springer-Verlag, New York, 1983.

20. J. L. Díaz-Barrero, M. Grau-Sánchez and P. G. Popescu, Refinements of Aczél, Popoviciu and Bellman's inequalities, Comput. Math. Appl., 56(9) (2008), 23562359.

\author{
Shanhe Wu \\ Department of Mathematics and Computer Science, \\ Longyan University, \\ Longyan, Fujian 364012, \\ P. R. China \\ E-mail: wushanhe@yahoo.com.cn
}

